\title{
PharmaSpy: um sistema de apoio a área de Farmacovigilância
}

\author{
Rian Alves ${ }^{1}$, João Dias ${ }^{1}$, Lina Garcés ${ }^{2}$, Vânia Neves ${ }^{3}$ \\ ${ }^{1}$ Departamento de Ciência da Computação, Universidade Federal de Juiz de Fora. \\ ${ }^{2}$ Instituto de Ciências Matemáticas e Computação, Universidade de São Paulo. \\ ${ }^{3}$ Instituto de Computação, Universidade Federal Fluminense \\ \{rian.alves, jpdias\}@ice.ufjf.br, linamgr@icmc.usp.br, vanialic.uff.br
}

\begin{abstract}
Medicaments adverse effects result from different factors. Pharmacovigilance is the health area responsible for the collection, detection, assessment, monitoring, and prevention of medicaments side effects, and also they audit medicaments production by pharmaceutics. We present PharmaSpy, an IS which aims to support ANVISA on its auditing processes at Brazil, decreasing risks for patients.

Resumo. Reações adversas a medicamentos podem ser geradas por diferentes fatores. A farmacovigilância é a área responsável por coletar, detetar, avaliar, monitorar, e prevenir tais reações e vigiar que as farmacêuticas produzam medicamentos de qualidade. Neste trabalho éapresentado o PharmaSpy, um SI que visa apoiar os processos de farmacovigilância no Brasil, promovendo a auditoria de medicamentos feita pela ANVISA, e diminuindo os riscos para os pacientes.
\end{abstract}

\section{Contextualização}

Apesar da qualidade dos medicamentos serem comprovadas dentro dos laboratórios farmacológicos, esses medicamentos podem apresentar efeitos adversos nos pacientes devido a diferentes tipos de erros presentes durante sua produção, identificação, prescrição, administração, entre outros [Carvalho et al. 1999]. A farmacovigilância é uma área da saúde que é responsável por garantir a qualidade dos medicamentos e prevenir seus riscos aos pacientes devido a efeitos adversos. Especificamente no Brasil, a indústria farmacêutica é regulada pela ANVISA ${ }^{1}$, porém essa agência não conta com um sistema de informação (SI) integrado para apoiar seus processos de regulação, sendo a maior parte desses processos realizados mediante comunicação telefônica.

A utilização desses sistemas de informação facilita o gerenciamento de conhecimento e de processos bem como a tomada de decisões nas organizações [A. G. Rosseti 2007]. Na área de farmacovigilância podem auxiliar na identificação e resolução de tais problemas, sendo possível controlar requisições, gerar estatísticas e desenvolver ações públicas para identificação de efeitos adversos dos fármacos, identificação de problemas em lotes ou fabricantes, monitoramento de vendas ilegais, entre outras adversidades referentes a qualidade dos produtos fármacos. Sendo assim, os SIs auxiliam as organizações responsáveis não só na identificação dos problemas como também na resolução deles. Uma vez que esses problemas são identificados, ações para a prevenção ou minimização de danos à saúde dos usuários podem ser tomadas.

\section{Sistema PharmaSpy}

Para aumentar a quantidade e qualidade das informações na área de farmacovigilância, este trabalho propõe o sistema PharmaSpy que auxiliará na captação de dados e informações sobre medicamentos vendidos utilizando da multidão de usuários (crowdsourcing) e farmácias.

\footnotetext{
${ }^{1}$ Agência Nacional de Vigilância Sanitária
} 
O objetivo é possibilitar que a população reporte efeitos adversos dos medicamentos, problemas relacionados a validade dos fármacos, distribuição e mantenimento. O sistema permitirá o cadastramento de três tipos de usuários, sendo eles: pacientes, profissionais da área da saúde, e servidores da ANVISA. Cada um deles tem acesso a um nicho do sistema e consequentemente a serviços e informações diferentes. Os pacientes serão capazes de reportar efeitos adversos e problemas relacionados ao medicamento adquirido por meio da abertura de chamados. Profissionais da saúde serão capazes de reportar adversidades envolvendo os medicamentos por ele manipulados, administrados, prescritos, ou adquiridos, podendo também anexar fotos do medicamento, notas fiscais, e informar histórico de compras e problemas. Usuários da ANVISA podem fazer uso do PharmaSpy gerando relatórios, visualizações, e informes dos mais variados tipos e que sejam de interesse para melhorar seus processos de farmacovigilância. Além disso, esses usuários têm acesso às denúncias e solicitações realizadas pelos usuários que relatam os efeitos adversos. Para melhorar a visualização e percepção das informações disponibilizadas pelos demais usuários, que fazem parte da multidão de consumidores de medicamentos regulados pela agência, o PharmaSpy exibirá elementos gráficos, indicadores, e tabelas.

Pretende-se que o PhamaSpy seja um sistema online com design responsivo permitindo, dessa forma, que o usuário o acesse de qualquer dispositivo, seja mobile ou não. Ele ainda apresenta menus e acessos interativos para facilitar a utilização das funcionalidades e, com isso, possibilitando alcançar um público que possa apresentar dificuldades, como no caso de idosos.

\section{Contribuições do Sistema}

A utilização do PharmaSpy contribuirá para identificação de produtos e seus lotes que podem causar danos a saúde da população. A partir das informações geradas, responsáveis da ANVISA podem tomar decisões e elaborar ações para minimizar ou até mesmo inibir os problemas relacionados às adversidades provocadas pelos produtos fármacos, sua distribuição e/ou seu armazenamento. Podem ser realizadas, por exemplo, ações de prevenção, acréscimo de observações relacionadas ao uso, suspensão do medicamento ou de algum de seus lotes, e ainda identificação de possíveis indivíduos que tentam burlar as leis impostas pela farmacovigilância brasileira.

\section{Conclusões e Perspectivas Futuras}

Espera-se que com a utilização desse sistema seja possível aumentar a qualidade das informações para melhorar o processo regulatório da produção e prescrição de medicamentos no Brasil, bem como auxiliar a tomada de decisões referentes a prevenção de danos a saúde dos consumidores.

\section{Referências}

A. G. Rosseti, A. B. T. M. (2007). O papel da tecnologia da informação na gestão do conhecimento. In 2007 Ci. Inf., Bras'ilia, volume 36, pages 124-135.

Carvalho, V. T. d., Cassiani, S. H. D. B., Chiericato, C., and Miasso, A. I. (1999). Erros mais comuns e fatores de risco na administração de medicamentos em unidades básicas de saúde. Revista Latino-Americana de Enfermagem, 7:67 - 76. 